\title{
Innovative last mile delivery concepts: Evaluating last mile delivery using a traffic simulator
}

\author{
Dylan Johnson \\ Energy Institute \\ University College London \\ London, United Kingdom \\ dylan.johnson.19@ucl.ac.uk
}

\author{
Emmanouil Chaniotakis \\ Energy Institute \\ University College London \\ London, United Kingdom \\ m.chaniotakis@ucl.ac.uk
}

\begin{abstract}
This paper investigates, through a simulation approach, how novel alternative last-mile solutions (LMS) can harmonise network efficiency with environmental sustainability in a Washington D.C. urban setting. The ability of public buses to scale delivery services with demand was fundamental to reducing Greenhouse Gas (GHG) emissions and harmful air pollutants. Extending the network by increasing the number of delivery points, this paper employed a K-means clustering algorithm to determine optimal locations of Urban Consolidation Centres (UCCs). Utilising UCCs enabled further environmental and efficiency gains to be realised through consolidation and the ability to deliver "very last mile" through E-cargo bikes.
\end{abstract}

Index Terms-Crowd Logistics, Public Transport Piggybacking, Last Mile Delivery, Urban Consolidation Centres

\section{INTRODUCTION}

Responsible for over 24\% of global Carbon Dioxide (CO2) emissions and over $14 \%$ of annual GHG emissions, the global transport sector must decarbonise rapidly if global climate change targets are to be met [1]. However, at a time when global GHG emissions must fall, transport emissions are increasing, as efficiency gains are being more than offset by greater volumes of travel [2]. In terms of transport modes, $72 \%$ of global emissions are derived from road vehicles with passenger road transport contributing to 3.6 Gigatons of Carbon Dioxide $(\mathrm{GtCO} 2)$ and freight vehicles accounting for 2.4 GtCO2 [1]. Within freight transportation, the last mile problem (LMP), the last leg of the delivery service, is consistently the most expensive and one of the highest polluting segments of the supply chain [3]. Last-mile delivery (LMD) has increasingly received attention as the unprecedented growth in ecommerce has seen e-commerce sales triple globally between 2014-2019; placing considerable stress on city planners and businesses to deliver rapid, low-cost and sustainable last-mile services [4].

Lately, to satisfy growing consumer demand for products online, the number of delivery vehicles in the top 10 most populated cities globally could increase by $36 \%$ by 2030 [5]. Driven by expected global growth in purchasing power, increased urbanisation, the emergence of further instant-delivery

978-1-7281-8995-6/21/\$31.00 @2021 IEEE digital business models and technological advancements, demand for urban LMD services is forecasted to grow by $78 \%$ by 2030 [5]. This demand surge for rapid, on-demand deliveries is expected to induce a $21 \%$ rise in urban traffic congestion and contribute to a $30 \%$ rise in emissions to 25 million tonnes of CO2 per year from LMD by 2030 [5]. Furthermore, enforced lockdowns bought about by the COVID-19 pandemic have caused online shopping sales to increase dramatically. In the United States May 2020 exhibited a 78\% increase over May 2019, and sales in April and May were 7\% higher than in November and December 2019, the standard peak shopping period [6]. While the long-term impacts of the of COVID-19 remains uncertain, it is likely to have accelerated this trend towards home delivery [7].

Currently, practices that attempt to circumvent the perceived trade-off between urban LMD efficiency and environmental sustainability prioritise vehicle electrification and off-peak delivery. However, frequently neglected are schemes that favour the integration of freight and passenger transportation. "Crowdshipping", "Freight-Sharing", or "Crowd logistics" are all concepts that aim to exploit the underutilised capacity in various passenger transportation modes to additionally deliver goods. In theory, significant economic and environmental gains in sharing urban infrastructure exist. Most fundamentally, sharing unused vehicle capacity would reduce the number of necessary trips and alleviate congestion [8]. At the same time, integrating people and cargo can make on-demand transport options more economical, flexible and reliable for businesses and consumers [9]. Yet, the overall GHG reductions from future delivery frameworks will greatly depend on how pure freight trips are replaced [10]. With the necessary infrastructure modifications and stakeholder support, freight trips could be substituted by public transport, walking or bicycle trips and have low environmental and congestion impacts. However, freight trips, replaced by private, motorised vehicles may induce rebound effects, adding trips and increasing negative externalities [11]. Hence, given how last-mile logistics are changing both rapidly and unpredictably to satisfy consumers desire for on-demand delivery, it is imperative that transport modellers produce quantitative indications of the network/environmental effects across varying scenarios. 


\section{LiteRATURE REVIEW: THE SHARED ECONOMY \& LAST} MILE LOGISTICS

\section{A. Crowd Logistics}

Crowd Logistics (CL) harnesses information technology to effectively provide delivery services more efficiently than traditional logistic services. Today CL business models encompass a variety of services including storage innovations, long-distanced freight shipping/forwarding, however none has gathered more investor or academic attention than its role in LMD [12]. CL incorporates various stakeholders: the suppliers who supplies the good, "the crowd" that physically completes the delivery, the customer who collects the final shipment, and the digital platform that coordinates the operation [13].

Economically, the benefits are rationalised through reduced costs and increased flexibility for businesses, carriers and consumers [14]. Commonly accepted within the literature is how necessitating digital platforms instead of asset-heavy infrastructure enable logistic companies to greatly reduce costly investments in vehicle fleets and employees [12]. Such cost reductions are likely to be shared with the consumer thereby benefiting consumers in the form of lower prices and greater utility [15]. Similarly, benefits could arise from greater flexibility in both the timings of when the deliveries occur and in the quantity of deliveries, potentially providing a more available, more frequent and customised service to consumers [12]. Lastly, CL can yield extensive benefits to carriers. Existing drivers for traditional logistics companies could use their spare capacity on their return trip as an additional earning opportunity [16]. Furthermore, employing the community, "the crowd" benefits from increased flexibility in having additional income opportunities by participating as a driver/carrier whenever convenient for them [15].

From a sustainability perspective, CL has the potential to significantly reduce emissions from LMD. Novel research often concludes that through exploiting existing trips and increasing vehicle loads, CL cuts the amount of network trips, thus reducing congestion and air polluting emissions [17]. Further environmental gains can be realised through a reduction of failed deliveries as crowd-sourced deliveries are more likely to be delivered when the recipient is present [13]. Yet, the literature lacks a nuanced analysis of the additional potential negative externalities brought about by CL. A Finnish case-study on crowd-sourcing library deliveries highlighted that in practice the rebound effects of drivers traveling extra distances motivated by monetary compensation, can significantly reduce environmental improvements [18]. Similarly [11], whose investigation finds a paradox: the greater the success of the CL and the higher monetary compensation to drivers, the greater the rebound effect and hence the social and environmental consequence.

\section{B. Public Transport Piggybacking}

Public transport services (PTS) are generally fast, economical, reliable and offer robust coverage of urban centres, however, notably experience periods of unprofitable and offpeak periods. Given how such qualities are shared with the needs of LMD, incorporating freight services into public transport infrastructure is an attractive solution for businesses and public transport services. While in practice most initiatives of integrating public transport with last mile logistics harness either buses or trams, the majority of academic studies focus on the use of metro systems. Early work has concentrated on the conceptual model of such multi-modal supply chains. A theoretical ULS dependent on public transport networks requiring depots, a metro system, and delivery servicemen was devised; feasibility issues including costliness and security were highlighted [19]; the importance of infrastructure at metro stops that can facilitate storage and re-distribution was stressed [20]; and the potential negative public opinion of such schemes was emphasised [21]. However, more contemporary works have disputed how passengers may disapprove of such initiatives. For instance, a Japanese pilot project which transported goods in the Sapporo city centre, found the scheme could effectively substitute conventional truck transportation while being favourably supported by the general public [22].

Few papers propose quantitative methods to evaluate the viability of metro-integrated logistics systems for intra-city delivery. In investigating the effects of piggybacking freight on roadside causalities in Newcastle-upon Tyne, the paper monetised the value of reduced roadside accidents and found significant monetary savings that more that cover the needed infrastructure changes at metro stations [23]. An investigation into a rail transport system in Paris carried out by the supermarket chain Monoprix, a reduction of 700,000 lorry miles, resulting in a $36 \%$ decrease in particulates emitted, $56 \%$ decrease of nitrous oxides and a 47\% in C02 emissions was calculated [24]. However, attributable to the extra handling, low volume per delivery and uneven distribution of freight flow transporting via metro was more expensive than conventional truck distribution. Additionally as Monoprix employed passenger rail facilities during off-peak times i.e. overnight, residents in surrounding areas complained of increase noise pollution during unsociable hours.

\section{Urban Consolidation Centres and Public Transport with Crowd Logistics}

For public transport services to effectively act a LMD service, either the recipient or storage infrastructure should be present at the public transport station (PTS). Instead, due to the inconvenience of collection or anxiety towards smart-lockers, there will likely always be demand for home delivery or a "very last-mile" delivery [24]. Thus, increasingly researchers have engaged with the possible duel-functionality of PTSs, serving both passengers and freight. The integration of multi-modal urban distribution centres at train stations was shown to enable low emission vehicles to deliver fish for minimally distanced last leg trips [25]. While the scheme was not economically competitive with conventional truck delivery, there was substantial emission savings. Therefore the work recommended that governments support economically the creation of UCCs to provide incentives for innovative green LMD. 


\section{Simulation Design \& Formulation of Routing PROBLEM}

A. Washington D.C based Network

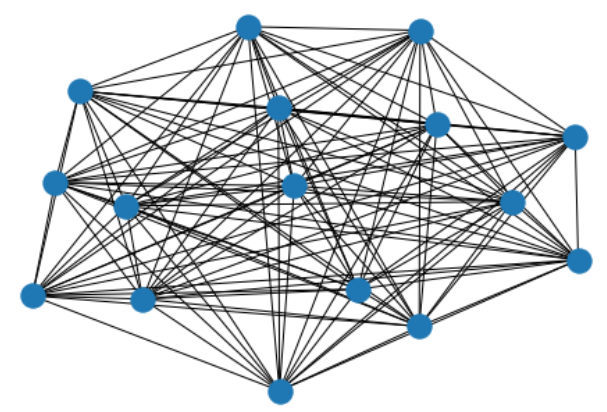

Fig. 1: Eigenspace graphical projection of network

We select our desired network by exporting a small section of northwestern Washington D.C from Open Street Maps. The network is characterised by a grid street plan with the majority of streets being either single or dual carriageways. We assume our network to have the following salient features: 15 randomly placed delivery locations, 1 depot, 2 Bus Routes: West line (9 stops), East line (7 stops). To illustrate, a network graph projecting the edges (euclidean distances) between all the nodes (delivery locations and depot) are displayed above in Fig 1. Each bus and delivery stop has a stop duration of 20 simulation seconds to account for the period necessary to complete a delivery, and each bus route is completed a minimum of 25 times in the simulation. A major assumption of our network is that the majority of bus stop are conveniently positioned close to our delivery locations. Thus this represents a situation where metro authorities are purposed to service to both passenger and freight demand.

\section{B. Simulations in Sumo}

"Simulation of Urban MObility" (SUMO) is an open source, microscopic and continuous traffic package that allows modelling of inter and multi modal traffic systems. To run simulations on Sumo, a network file consisting of edges and nodes, a route file including trips information, and an additional file which details the location and size of bus and delivery stops, is required. The investigation randomly allocates locations for the depot and delivery stops. Given their locations a Manhattan Distance Matrix is calculated to enable the delivery routes to be optimised. These routes are then optimised through solving a capacitated vehicle routing problem (CVRP). Next, the optimal delivery routes are combined with the network congestion to create the final route file. Finally, together with the network and bus and delivery stop locations, SUMO simulates the traffic system to yield outputs on both trip information and emissions.

\section{Scenarios}

(i) Reference Scenario

a) Purpose: Represent conventional LMD

b) Route: Depot $\rightarrow$ Delivery Points $\rightarrow$ Depot c) Vehicle Type: Delivery Vans

d) Abbreviation: BC (Base Case)

(ii) Crowd Logistics Return

a) Purpose: Present CL with feedback effects

b) Route: Depot $\rightarrow$ Delivery Points $\rightarrow$ Depot

c) Vehicle Type: Cars and Motorbikes

d) Abbreviation: $\mathrm{CL}(\mathrm{R})$

(iii) Crowd Logistics

a) Purpose: Highlight optimal CL model

b) Route: Depot $\rightarrow$ Delivery Points

c) Vehicle Type: Cars and Motorbikes

d) Abbreviation: CL

(iv) Public Transport Piggybacking

a) Purpose: Integrate delivery into passenger services.

b) Route: Depot $\rightarrow$ Bus Stop $\rightarrow$ Delivery Points

c) Vehicle Type: Metro-bus and Car

d) Abbreviation: PT

(v) Public Transport piggybacking with Crowd Logistics

a) Purpose: Extend the PT scenario and present a LMD service where UCCs enable crowdshipped very LMD

b) Route: Depot $\rightarrow$ Bus Stop $\rightarrow$ Delivery Points

c) Vehicle Type: Bus and E-cargo Bike

d) Abbreviation: PT + CL

\section{Capacitated Vehicle Routing Problem (CVRP)}

The vehicle routing problem finds the optimal set of routes that a vehicle fleet should traverse to deliver a set of goods to a set of customers. From graph theory, the CVRP can be represented by a directed graph $G(E, V)$, where $V=$ $0,1, \ldots, n$ represents the set of nodes and $\mathrm{E}$ is the set of edges. The depot is noted as node $j=0$, and delivery locations are nodes $j=1,2, \ldots, n$. Each edge represents a route from node $\mathrm{i}$ to node $\mathrm{j}$. The weight of each edge $C_{i, j}>$ 0 corresponds to the cost of going from node i to node j. $x_{\text {rij }}$ is the binary decision variable that indicates whether vehicle $r, r \in\{1, \ldots, p\}$ traverses an edge $(i, j)$ is part of the solution. Thus, extending the seminal Dantzig, Fulkerson and Johnson TSP formulation, we can express the CVRP formally [26]:

$$
\min \sum_{r \in p} \sum_{i \in V} \sum_{j \in V} C_{i, j} x_{r, i, j}
$$

s.t

$$
\begin{array}{lr}
\sum_{r=1}^{p} \sum_{i=0, i \neq j}^{n} x_{r, i, j}=1, & \forall j \in\{1, \ldots, n\}, \\
\sum_{j \in 1}^{n} x_{r, 0, j}=1, & \forall r \in\{1, \ldots, p\}, \\
\sum_{i=0, i \neg j}^{n} x_{r, i, j}=\sum_{i=0}^{n} x_{r, j, i}, \forall j \in\{0, ., n\}, \forall r \in\{1, ., p\}, \\
\sum_{i=0}^{n} \sum_{j=1, i \neq j}^{n} d_{j} x_{r, i, j} \leq Q, & \forall r \in\{1, \ldots, p\},
\end{array}
$$




$$
\begin{aligned}
& \sum_{r=1}^{p} \sum_{i \in S} \sum_{j \in S, i \neg j} x_{r, i, j} \leq|S|-r(s), \quad \forall S \subseteq\{1, \ldots, n\}, \\
& x_{i j} \in\{0,1\}, \quad \forall r \in\{1, \ldots, p\}, i, j \in\{0, \ldots, n\}, i \neq j .
\end{aligned}
$$

\section{E. Delivery Demand Assumptions}

Consumers order products of different shapes, sizes and weights and thus this paper will assume that demand for delivery packages approximates to a log normal distribution, with $\log$ mean 1.2 and $\log$ standard distribution 1.2. We assume a log-normal distribution for our delivery weight due to its zero lower bound and is skewed right, both of which characterise low-weight deliveries [27]. To obtain the weight of each delivery package we generate 75 random numbers according to our distribution. Since the initial network has 15 delivery stops we create a vector of the first 15 numbers within that list of numbers. Next, we assume 4 deliveries at each of the delivery stops at every one time, so we sum our vector by the three additional vectors of length 15 . A pre-determined quantity of those deliveries is defined for the simulation and there exists four scenarios in which that demand scale factor changes. Table I presents such demand scenarios along with the resulting total weight of deliveries for that scenario.

TABLE I: Delivery Demand Scenarios

\begin{tabular}{|c|c|c|c|}
\hline Demand & $\begin{array}{l}\text { Delivery Weight } \\
\text { Per Cycle }(\mathrm{kg})\end{array}$ & Scale Factor & $\begin{array}{l}\text { Total Delivery } \\
\text { Weight }(\mathrm{kg})\end{array}$ \\
\hline Low & 447 & x 15 & 6707 \\
\hline Mid - Low & 447 & x 25 & 11178 \\
\hline Mid - High & 447 & x 35 & 15650 \\
\hline High & 447 & x 45 & 20121 \\
\hline
\end{tabular}

\section{F. Vehicle Capacity Assumptions}

TABLE II: Capacity Assumptions for Vehicles

\begin{tabular}{|c|c|c|}
\hline Vehicle & Max Capacity (kg) & Assumed Capacity (kg) \\
\hline Cargo Bike & 70 & 34 \\
\hline Motorbike & 15 & 15 \\
\hline Car & 300 & 100 \\
\hline Box Van & 400 & 300 \\
\hline
\end{tabular}

TABLE III: Capacity Assumptions for MetroBus

\begin{tabular}{|c|c|c|}
\hline Attribute & Value & Unit \\
\hline Average Bus Capacity & 50 & People \\
\hline Assumed Space for freight & 5 & $\%$ \\
\hline Max Metro Bus Capacity & 4125 & $\mathrm{~kg}$ \\
\hline Realistic Metro Bus Capacity & 206 & $\mathrm{~kg}$ \\
\hline
\end{tabular}

As LMD services are reliant on the crowd, assuming a vehicles to have identical vehicle capacities is unrealistic. Hence, normal distributions around the vehicle's respective mean capacity are assumed.

\section{G. Sensitivity of MetroBus Capacity}

To evaluate the sensitivity of our results to MetroBus freight Capacity we will simulate for 3 levels of capacity, 5\%, 6\% and $7 \%$. To model this, we first calculate the number of bus trips required to satisfy peak demand given the specified capacity. From our log-normally distributed generated demand, we have demand for each delivery location. Those from locations serviced by MetroBus and by other means are as follows:
TABLE IV: Delivery Demand by Delivery Mode

\begin{tabular}{|c|c|c|c|c|}
\hline Delivery Source & $\begin{array}{c}\text { East } \\
\text { Bound }\end{array}$ & $\begin{array}{c}\text { West } \\
\text { Bound }\end{array}$ & $\begin{array}{c}\text { Crowd } \\
\text { Logistics }\end{array}$ & Total \\
\hline Demand per cycle $(\mathrm{kg})$ & 170 & 176 & 101 & 447 \\
\hline Peak Demand Total $(\mathrm{kg})$ & 7636 & 7940 & 4545 & 20121 \\
\hline
\end{tabular}

Given such, we can calculate the number of bus trips needed to satisfy peak demand at each level of capacity:

TABLE V: Required Bus Cycles

\begin{tabular}{|c|c|c|c|}
\hline Available Capacity for Freight & $5 \%$ & $6 \%$ & $7 \%$ \\
\hline Capacity per bus (kg) & 206.25 & 247.50 & 288.75 \\
\hline Number of Cycles (East Bound bus) & 38 & 31 & 27 \\
\hline Number of Cycles (West Bound bus) & 39 & 33 & 28 \\
\hline
\end{tabular}

\section{H. Extension: "Very last-mile delivery"}

The network can be expanded to explore a general case where delivery services can use UCC services at PTSs. Here, we seek to identify a PTS as a suitable location for a UCC and subsequently find an optimal route for a CL service or courier service.

To define our preferred UCC location we use a K-means clustering algorithm. We run a k-means algorithm descending from 6 clusters, and select the optimal number of clusters based on the "elbow" method. Indicating an optimal of 3 clusters, the bus stations with the minimum distance from the centroid of our two new clusters are selected as the UCCs. It is assumed that our original depot will continue to act as the distribution centre for those delivery locations within its cluster.

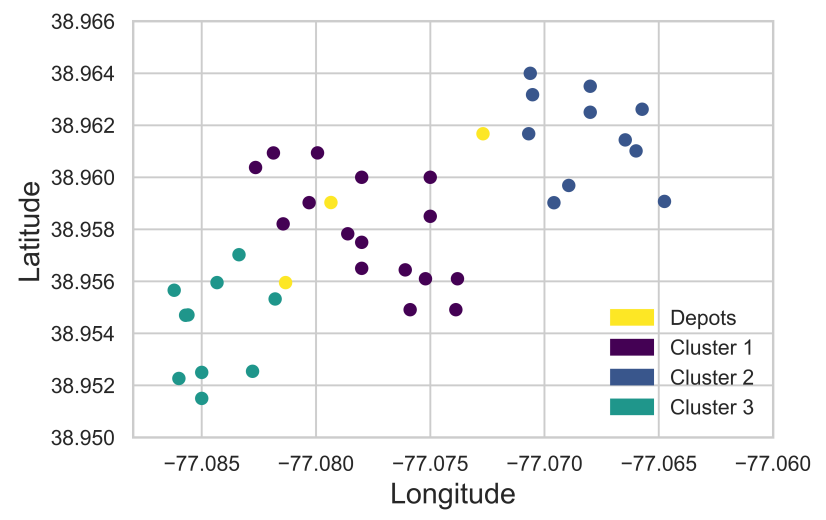

Fig. 2: Clustered Delivery Locations with Depot Locations

From the UCCs, 2 "very last mile" solutions exist:

(i) The delivery is done by a low emission/capacity vehicle and the within-cluster routes are obtained by a TSP.

(ii) The delivery takes the no return CL scenario.

To measure the relative impact of these alternative logistics systems, we use a methodology comparable to our reference scenario solved with a CVRP to act as the counterfactual. 


\section{ANALYSIS \& RESUlTS}

\section{A. Capacitated Vehicle Routing Problem}

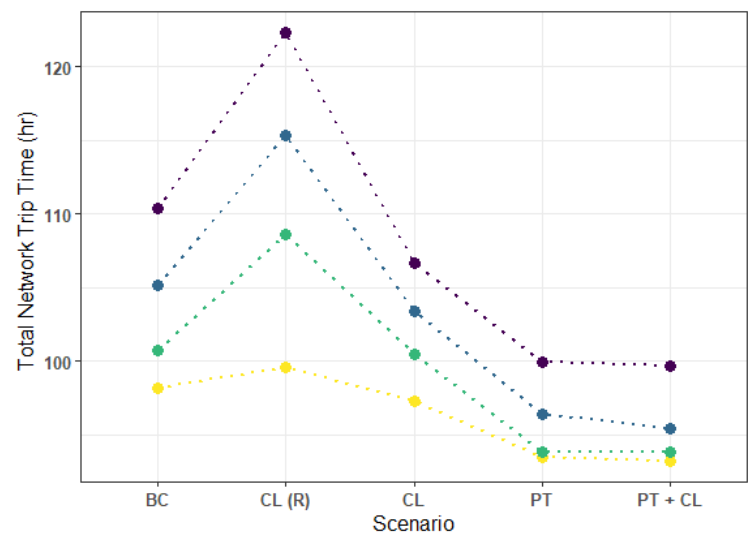

Fig. 3: Network efficiency effects of Scenarios

With congestion fixed and demand scaled demand by 25 , 35 and 45 , the BC scenario induces $0.20 \%, 1.71 \%$ and $3.52 \%$ greater total trip time than when compared to CL. Though increasing the quantity of delivery trips, the benefit of vehicles characterised by faster acceleration and break speeds on network efficiency are evident. However, such efficiency benefits of a CL LMS are eliminated if crowd-shippers return to the depot. While the CL (R) scenario induces the greatest total network trip time, duration increases consistently rather than at increasing rates. This indicates that this is primarily due to pure quantity of trip increases rather than inducing further consequential knock-on congestion. The PT and PT + CL Scenarios both considerably outperform BC in terms of network efficiency across all demand scales with negligible differences between the two scenarios.

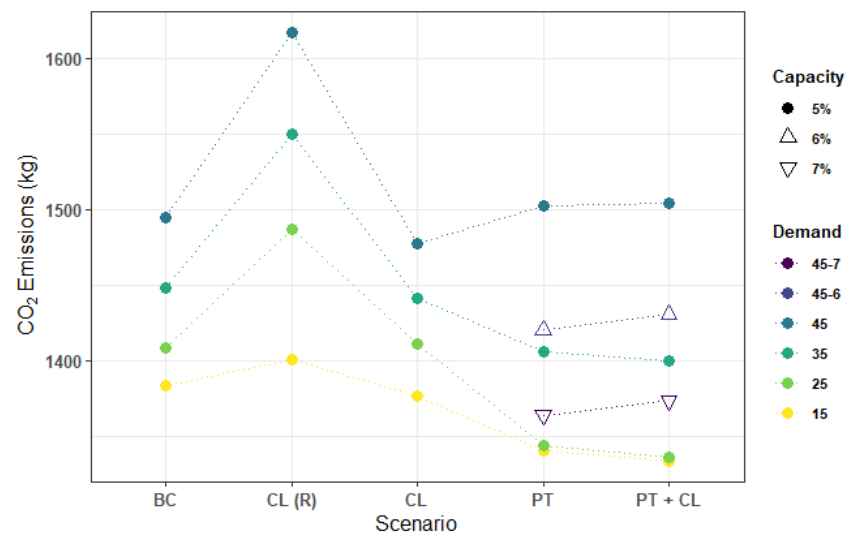

Fig. 4: Emissions with Bus Capacity

At the highest level of delivery demand, LMS requiring additional bus services underperform both BC and CL scenarios (Fig. 4). This emission elasticity to demand is largely driven by the high $\mathrm{CO}_{2}$ emitting nature of public buses. Therefore solutions that mitigate this sensitivity need to exist for piggybacking on public buses to be considered an economically viable green LMS. Here we investigate how increasing the assumption that $5 \%$ of bus capacity can be used for freight to $7 \%$. The results are significant, elucidating that increasing available freight capacity by $2 \%$ can yield a $5.4 \%$ decrease in $\mathrm{CO} 2$ emissions when compared to the $\mathrm{BC}$.

However, while freight capacity may be scalable with freight demand on PTS during off-peak hours, can we assume this to be the case when passenger demand is high? The COVID-19 pandemic has dramatically changed, and is expected to continue to change, travel habits for the foreseeable future. While the metro authority expects passenger demand to increase up to socially distanced capacity legally enforced capacity limits combined with behavioural factors are likely to limit the demand rebound.

B. "Very last mile" Delivery

TABLE VI: "Very Last Mile" Simulation Results

\begin{tabular}{|c|c|c|c|c|}
\hline & Unit & BC & PT + CL & PT + TSP \\
\hline C02 & $\mathrm{kg}$ & 1856 & 1780 & 1713 \\
\hline NOx & $\mathrm{kg}$ & 3.01 & 3.01 & 2.98 \\
\hline Total Trip Duration & $\mathrm{mins}$ & 127.44 & 121.4 & 128.4 \\
\hline
\end{tabular}

Environmentally, the BC is the undesirable LMS, failing to save trip distance from PT services, consolidate its delivery freight and switch to lower emitting vehicles at an UCC. These factors culminate in a $8.35 \%$ and $167.7 \%$ increase in $\mathrm{CO} 2$ and $\mathrm{HC}$ emissions relative to the PT + TSP scenario. Furthermore our PT + TSP scenario outperforms the PT + CL scenario. This is rationalised though the PT + TSP employing E-cargo bikes instead of cars or motorbikes [10]. In terms of network efficiency, the PT + CL scenario is the preferable LMS. The PT + CL scenario results in $5.45 \%$ decreases in total network trip time when compared to TSP, and a $4.74 \%$ fall in total trip time to BC. This indicates possible efficiency gains in CL methods that use low capacity vehicles with efficient acceleration and deceleration capabilities in areas of high delivery density.

\section{UCC street level analysis}

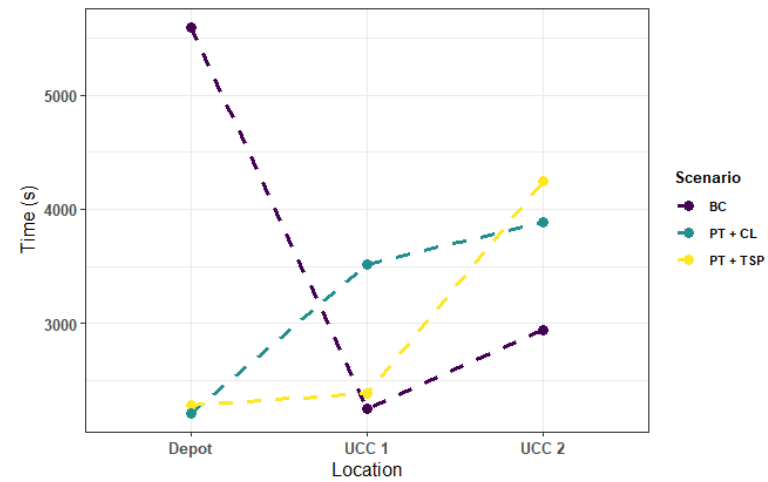

Fig. 5: Vehicle Time spent on edge with a distribution centre

Fig 5 elucidates the total time that vehicles were present on the corresponding edge of a depot across each scenario. Moving from a mono-depot logistics system to a multi-depot system, the concentration of traffic around the original depot is reduced by $59.18 \%$ and $60.4 \%$ as its operational frequency declines. However as operational UCCs are introduced, vehicle time spent around UCCs increases in all four cases. PT 
scenarios have three highly congested areas and the simulation results indicate decreases in total time spent around UCCs in these scenarios. The increased quantity of delivery vehicles in the CL scenario and the greater assumed loading time were the main factors why UCCs in PT+CL experienced greater congestion than in PT+TSP.
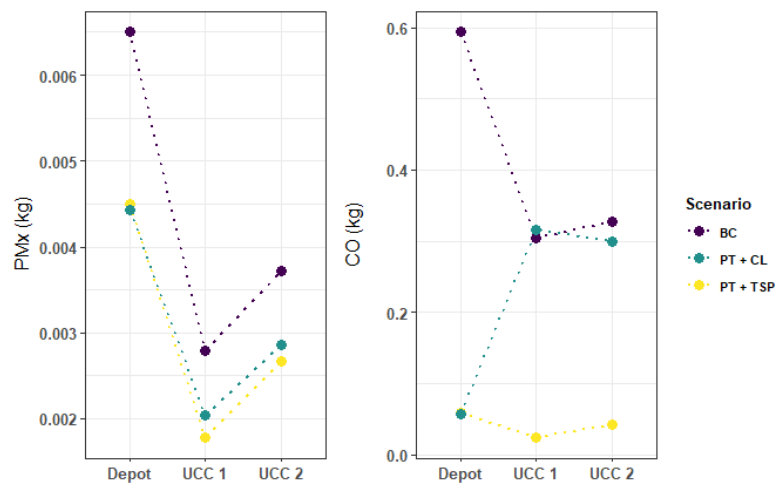

Fig. 6: Excess Capacity on Bus Services

Rather than increasing the harmful pollutants both air quality metrics fall in quantity emitted (Fig 6.) This is realised through those additional vehicles originating from these UCCs being low-emitting, low capacity vehicles as these very last mile trips are frequent, short-distanced and low-weight. The reference scenario maintains higher levels of $\mathrm{CO}$ and $\mathrm{PMx}$ emissions because the vans are still required to delivery to those delivery locations on the same street as the UCCs.

\section{Conclusions, Recommendations \& Future Work}

We found two innovative LMS that successfully harmonise network efficiency with environmental sustainability. These LMS however only satisfied such objectives given certain necessary features. The 'piggybacked' LMS required freight capacity to be scaleable with delivery demand while crowdlogistics required minimal feedback effects. Extending our network to allow for the possibility of UCCs we found further environmental and efficiency gains to realised through consolidation and the consequential ability to deliver complete "very last mile" through E-cargo bikes. Moreover, while there was evidence of increased congestion around the UCCs, the total vehicle time around all network depots decreased.

\section{ACKNOWLEDGMENT}

The research reported in this paper has been supported by European Union's Horizon 2020 research and innovation programme under Grant Agreement No 815269, project HARMONY and UCL BSEER Development funds (RELM project)

\section{REFERENCES}

[1] IEA. (2020) Transport sector co2 emissions by mode in the sustainable development scenario, 2000-2030. https://www.iea.org/dataand-statistics/charts/transport-sector-co2-emissions-by-mode-in-thesustainable-development-scenario-2000-2030.

[2] S. Wang and M. Ge. (2019) Everything you need to know about the fastest-growing source of global emissions: Transport. https://www.wri.org/blog/2019/10/everything-you-need-know-aboutfastest-growing-source-global-emissions-transport.
[3] J. Brown and A. Guiffrida, "Carbon emissions comparison of last mile delivery versus customer," International Journal Of Logistics Research And Applications, vol. 17(6), pp. 503-521, 2014.

[4] eMarketer. (2019) Transport sector co2 emissions by mode in the sustainable development scenario. https://www.emarketer.com/content/ global-ecommerce-2019.

[5] WEF. (2020) The future of the last-mile ecosystem. http://www3 weforum.org/docs/WEF_Future_of_the_last_mile_ecosystem.pdf.

[6] eMarketer. (2020) Us ecommerce will rise $18 \%$ in 2020 amid the pandemic. https://www.emarketer.com/content/ us-ecommerce-will-rise-18-2020-amid-pandemic?ecid=NL1001.

[7] A. Unnikrishnan and M. Figliozzi, "A study of the impact of covid-19 on home delivery purchases and expenditures," Working Paper, 2020.

[8] J. Miller, Y. Nie, and A. Stathopoulos, "Crowdsourced urban package delivery: Modeling traveler willingness to work as crowdshippers," Transportation Research Record, vol. 2610(1), p. 67-75, 2017.

[9] Punel, Ermagun, and Stathopoulos, "Carbon emissions comparison of last mile delivery versus customer pickup," International Journal of Logistics Research And Applications, vol. 17(6), pp. 503-521, 2014.

[10] M. Simoni, E. Marcucci, V. Gatta, and G. Claudel, "Potential lastmile impacts of crowdshipping services: a simulation-based evaluation," Transportation, vol. 47, p. 1933-1954, 2020.

[11] W. Qi, L. Li, S. Liu, and Z. Shen, "Shared mobility for last-mile delivery: design, operational prescriptions and environmental impact," Manuf Serv Oper Manag, vol. 20(4), p. 737-751, 2018.

[12] V. Carbone, A. Rouquet, and C. Roussat, "The rise of crowd logistics: A new way to co-create logistics value," Journal of Business Logistics, vol. 38(4), pp. 238-252, 2017.

[13] H. Buldeo-Rai, S. Verlinde, and C. Macharis, "Shipping outside the box. environmental impact and stakeholder analysis of a crowd logistics platform in belgium," Journal of Cleaner Production, vol. 202, p. 806-816, 2018

[14] V. Demary and B. Engles, "Collaborative business models and efficiency - potential efficiency gains in the european union," 2016.

[15] S. Li, W. Wu, Y. Xia, M. Zhang, S. Wang, and M. Douglas, "How do crowd logistics platforms create value? an exploratory case study from china," International Journal of Logistics Research and Applications, vol. 22:5, pp. 501-518, 2019.

[16] R. Botsman, "Crowdshipping: Using the crowd to transform delivery," AFR Boss Magazine, 2014

[17] V. Gatta, E. Marcucci, M. Nigro, and S. Serafini, "Sustainable urban freight transport adopting public transport-based crowdshipping for b2c deliveries," Eur. Transp. Res. Rev., vol. 11(13), 2019.

[18] H. Paloheimo, M. Lettenmeier, and H. Warise, "Transport reduction by crowdsourced deliveries - a library case in finland," Journal of Cleaner Production, vol. 132, pp. 240-251, 2015.

[19] H. Liu, "Feasibility of using pneumatic capsule pipelines in new york city for underground freight transport." 2004.

[20] Y. Tan and Y. San, "Urban rail transit in low hours for express delivery," Urban Mass Transit, pp. 5-8, 2009.

[21] C. Liu, "Study on how to use beijing subways as a part of the city's logistics system in the evening and non-traffic peak time," Urban Stud, vol. 18, pp. 122-124, 2011.

[22] J. Kikuta, T. Ito, I. Yamamoto, and T. Yamada, "New subway-integrated city logistics system," Procedia - Social and Behavioral Sciences, vol. 39, pp. 476-489, 2012.

[23] A. Dampier and M. Marinov, "A study of the feasibility and potential implementation of metro-based freight transportation in newcastle upon tyne," Urban Rail Transit, vol. 1, pp. 164-18, 2015.

[24] L. Delaître and C. De-Barbeyrac, "Improving an urban distribution centre, the french case of samada monoprix," Procedia Soc Behav Sci, vol. 39, p. 753-769, 2012.

[25] A. Alessandrini, P. Delle-Site, F. Filippi, and M. Salucci, "Using rail to make urban freight distribution more sustainable," European Transport, vol. 50, pp. 1-17, 2012.

[26] Z. Borcinova, "Two models of the capacitated vehicle routing problem," Croatian Operational Research Review, vol. 8, pp. 463-469, 2017.

[27] TDBG. (2020) Delivery vehicles and containers. http://www.tdbg.de/en/ cu-service/delivery-vehicles-and-containers/.

The code used for this paper is publicly available here: https://github.com/DangerousDyl/Innovative_LM 